\title{
Multidisciplinary Management of Alport Syndrome: Current Perspectives
}

\author{
Clifford Kashtan \\ Department of Pediatrics, Division of \\ Pediatric Nephrology, University of \\ Minnesota Medical School, Minneapolis \\ MN, 55454, USA
}

\begin{abstract}
Alport syndrome is a multisystem disorder that universally affects the kidney and frequently involves the inner ear and the eye. Over the course of a lifetime, addressing the health care needs of a person with Alport syndrome and their family entails the services of primary providers, nephrologists, genetic counselors, audiologists, ophthalmologists, transplant physicians, kidney dieticians, and social workers as well as other healthcare professionals. This article attempts to provide context and guidance regarding the multidisciplinary care of Alport syndrome based on the natural history of the condition.
\end{abstract}

Keywords: Alport syndrome, collagen IV, genetic kidney disease, sensorineural hearing loss

\section{Introduction}

Alport syndrome is a genetic disorder of basement membrane collagen IV caused by pathogenic variants in the COL4A3, COL4A4 and COL4A5 genes. ${ }^{1}$ The COL4A5 gene is located on the $\mathrm{X}$ chromosome while the $C O L 4 A 3$ and $C O L 4 A 4$ genes reside on chromosome 2, resulting in three major genetic types of Alport syndrome $\mathrm{X}$-linked, autosomal recessive and autosomal dominant. The variants in the COL4A3, COL4A4 and COL4A5 genes disrupt the normal composition, structure and function of specific basement membranes in the kidney, inner ear (cochlea) and eye, resulting in progressive kidney disease, hearing loss and ocular anomalies in many affected individuals. Consequently, the medical journey of people with Alport syndrome involves multiple primary and subspecialty providers. The purpose of this monograph is to discuss the roles of care providers in the diagnosis and management of Alport syndrome.

\section{Clinical Natural History of Alport Syndrome}

The clinical manifestations of Alport syndrome, and the associated physical, emotional and financial burdens of the disorder, develop over time. In order to understand how physicians and allied professionals become involved in the care of people with Alport syndrome it is helpful to appreciate the temporal features of involvement of the kidney, cochlea and eye in people with Alport syndrome.

\section{Manifestations of Alport Syndrome in the Kidney}

The kidneys of newborn infants with Alport syndrome are grossly normal in structure, and the excretory, filtration and endocrine processes of the kidneys generally remain in the normal range for at least the first several years of life. However, at a molecular level the kidneys of these children are abnormal. Under
Correspondence: Clifford Kashtan

Email kasht00I@umn.edu 
normal circumstances the predominant form of collagen in glomerular basement membranes (GBM) is a scaffold composed of the collagen IV $\alpha 3, \alpha 4$ and $\alpha 5$ chains (the a345 collagen IV scaffold). In people with Alport syndrome, variants in the COL4A3, COL4A4 or COL4A5 chains, the genes respectively encoding the $\alpha 3, \alpha 4$ and $\alpha 5$ collagen IV chains, prevent or depress the normal deposition of the $\alpha 345$ collagen IV scaffold in GBM. Consequently, the predominant form of collagen in the GBM of people with Alport syndrome is a scaffold composed of $\alpha 1$ and $\alpha 2$ collagen IV chains (the $\alpha 112$ collagen IV scaffold). ${ }^{2,3}$ The replacement of the $\alpha 345$ collagen IV scaffold by the $\alpha 112$ collagen IV scaffold allows normal glomerular development in utero, but the $\alpha 112$ collagen IV scaffold is not sufficient to maintain the normal structural and functional properties of GBM over time, likely due to differences in molecular crosslinking that limit the strength of the $\alpha 112$ collagen IV scaffold, altered interactions with glomerular visceral epithelial cells (podocytes) and changes in intracellular signaling that promote the synthesis and anomalous deposition of certain laminins and other collagens (collagen V, collagen VI) and extracellular matrix proteins in the GBM. ${ }^{4,5}$ At the level of electron microscopy, the abnormalities of the $\alpha 345$ collagen IV scaffold are manifested initially as attenuation of the GBM. Over time there is progressive thickening and disorganization of the GBM, and gradual disappearance of the foot processes of glomerular podocytes. ${ }^{6}$

The earliest clinical finding in children with Alport syndrome is hematuria (blood in the urine). ${ }^{7}$ The hematuria may be macroscopic, resulting in tea- or cola-colored urine or diaper stains, but is more often microscopic, that is detectable only by microscopic examination or urine dipstick. Macroscopic hematuria is likely to lead to evaluation by a primary care provider and, at some point, referral to pediatric nephrology or urology. Microscopic hematuria may go undetected for years, since routine urinalysis is often not performed in children, at least in the United States. Microscopic hematuria may be detected incidentally, for example during evaluation of febrile illness, or may be revealed by intentional screening because of a known family history of Alport syndrome. Hematuria in people with Alport syndrome likely results from tiny ruptures in glomerular capillary walls, due to attenuation of the GBM. ${ }^{8}$

The kidney glomerulus is a selective filter that allows passage from blood to urine of large daily volumes of water and small solutes, but restricts the movement of plasma proteins, particularly those with a molecular weight above 60 kilodaltons, such as albumin. Selectivity of filtration relies on the complex organization of glomerular endothelial cells, GBM and glomerular visceral epithelial cells into a semipermeable barrier. This barrier breaks down as people with Alport syndrome age, allowing passage of albumin and other large proteins in quantities that exceed the absorptive capacities of the cells of proximal tubules, leading to elevated levels of albumin (albuminuria) and larger proteins (proteinuria) in the final urine.

Urinary albumin levels are estimated by the microalbumin-to-creatinine ratio and are normally very low (20 mg/gm creatinine or less). Microalbuminuria, defined as a urinary microalbumin-creatinine ratio greater than $30 \mathrm{mg} / \mathrm{gm}$ creatinine, is frequently detectable in children with Alport syndrome, and eventually progresses to overt proteinuria (defined as a urine protein-to-creatinine ratio greater than $0.2 \mathrm{mg} / \mathrm{mg}$ creatinine, corresponding to a microalbumin-creatinine ratio of about $200 \mathrm{mg} / \mathrm{gm}$ creatinine). ${ }^{9}$ Losses of protein via the kidneys may eventually exceed the synthetic capacities of the liver, leading to low serum albumin levels (hypoalbuminemia), salt and water retention and edema, which constitute the nephrotic syndrome.

The excretory function of the kidneys, that is the elimination of the daily burden of metabolic waste products, is normal in the early years of life in people with Alport syndrome. The excretory function of the kidneys is estimated by blood tests, including serum levels of creatinine and blood urea nitrogen, which increase as excretory function decreases. In people with Alport syndrome albuminuria and proteinuria precede any decline in excretory function, often by many years. Declining excretory function in people with Alport syndrome is associated with the replacement of normal kidney structures, the glomeruli and the tubules connected to the glomeruli, by scar tissue, in a process termed fibrosis. ${ }^{10}$ As in other chronic kidney diseases there are multiple drivers of fibrosis in the kidneys of people with Alport syndrome, at least some of which can be slowed or ameliorated by appropriate pharmacologic intervention. It is the fibrotic process in the kidneys that leads eventually to kidney failure - the need for dialysis or kidney transplantation - in people with Alport syndrome.

The pace of kidney disease progression in people with Alport syndrome is heavily influenced by the person's collagen IV genotype (the type of genetic variant) and, 
for the X-linked form of Alport syndrome (XLAS), by the person's sex chromosome complement (XX or XY). ${ }^{11,12}$ Genotype and sex chromosome complement also affect the severity of hearing loss and ocular changes in people with Alport syndrome, as will be discussed later.

In general, XY individuals with XLAS, who have a single abnormal copy of the COL $4 A 5$ gene, and people with autosomal recessive Alport syndrome (ARAS), who have variants in both copies of the COL $4 A 3$ or COL $4 A 4$ gene, have more rapid progression to kidney failure than XX individuals with XLAS, who have a normal copy of the COL4A5 gene as well as an abnormal copy of the gene, and people with autosomal dominant Alport syndrome (ADAS), who have a normal copy of the COL4A3 or $C O L 4 A 4$ gene as well as the variant copy of the gene.

In XY individuals with XLAS, the nature of the change in the COL4A5 gene is another variable that influences the age at which kidney failure develops. Variants that prevent the production of a full-length $\alpha 5$ collagen IV chain, such as deletion of large portions of the gene, or changes that disrupt the normal reading of the gene by the cell's nuclear machinery (nonsense variants), are associated with more rapid progression to kidney failure, compared with variants that change a single amino acid in the $\alpha 5$ collagen IV chain (missense variants). ${ }^{11,13,14}$ The nature of the COL4A5 gene variant does not influence kidney disease progression in $\mathrm{XX}$ individuals with XLAS, likely due to the much stronger effect of random X-chromosome inactivation (the process that determines which $\mathrm{X}$ chromosome is active in the cells of an XX individual). ${ }^{12}$ The effects of COL4A3 and COL4A4 genotype on kidney disease progression in people with ARAS and ADAS are less well understood and remain under investigation.

\section{Inner Ear Dysfunction in Alport Syndrome}

The $\alpha 345$ collagen IV scaffold is a critical component of basement membranes of the inner ear that contribute to normal perception and transmission of aural information to the brain. ${ }^{15,16}$ Absence or abnormal function of the $\alpha 345$ collagen IV scaffold in these inner ear basement membranes interferes with hearing through mechanisms that are still under investigation. Although the inner ear develops normally in utero in people with Alport syndrome and their hearing is normal at birth, sensorineural hearing loss is frequently detectable in childhood, especially in $\mathrm{XY}$ children with $\mathrm{X}$-linked disease and children with autosomal recessive Alport syndrome. As with the kidney disease of XLAS, the onset of hearing loss and the need for hearing aids occur earlier in XY individuals with COL4A5 variants that prevent the synthesis of a fulllength $\alpha 5$ collagen IV chain. ${ }^{11}$ The hearing loss typically first affects perception of high frequency sounds and over time increasingly involves frequencies associated with conversational speech. The hearing loss characteristically plateaus at a level that is amenable to amplification using hearing aids. XX individuals with XLAS can also experience hearing loss, although the risk is lower than in $\mathrm{XY}$ individuals. ${ }^{12}$ Hearing loss is relatively unusual in people with ADAS. ${ }^{17,18}$ Hearing loss in people with Alport syndrome is not improved by kidney transplantation.

\section{Eye Changes in Alport Syndrome}

The $\alpha 345$ collagen IV scaffold is normally found in several basement membranes of the eye, including the basement membrane of the cornea, the basement membrane surrounding the lens, known as the lens capsule, and the basement membrane of the retina. ${ }^{19-21}$ The eyes develop normally in utero and vision is typically unimpaired in children with Alport syndrome. The most prominent manifestations of the absence or dysfunction of the $\alpha 345$ collagen IV scaffold in the ocular basement membranes of people with Alport syndrome are anterior lenticonus, altered pigmentation of the retina and thinning of the retina. $^{22,23}$

Anterior lenticonus is a change in the shape of the lens that results from thinning and weakness of the lens capsule, the basement membrane that surrounds the semisolid lens and maintains its normal shape. Since anterior lenticonus has, so far, been found only in people with Alport syndrome, the finding of anterior lenticonus is diagnostic of the condition. Anterior lenticonus occurs in a portion of XY individuals with XLAS and people with ARAS. It can occur in XX individuals with XLAS but is extremely rare in people with ADAS. Like Alport kidney disease and hearing loss, anterior lenticonus is more likely to affect $\mathrm{XY}$ individuals with $C O L 4 A 5$ variants that preclude production of a full-length $\alpha 5$ collagen IV chain. In anterior lenticonus the central portion of the lens protrudes forward into the anterior chamber of the eye. Although anterior lenticonus can be detected by an experienced practitioner using an ophthalmoscope, it is typically found by an ophthalmologist by means of slit-lamp examination. The change in lens shape can alter the refraction of light images by the lens, resulting in the need for corrective 
lenses. Cataract formation is another potential complication of anterior lenticonus.

A characteristic alteration of the pigmentation of the retina is a common feature of XLAS and ARAS. ${ }^{22}$ Instead of a homogeneous pinkish-red hue, areas of the retina, particularly around the macula (the central area of the retina), display whitish or yellowish flecks, sometimes described as a dot-fleck retinopathy. The process that produces this change in pigmentation is not understood. The pigmentary alterations do not appear to cause any impairment of vision. Thinning of the retina can be observed in people with Alport syndrome using a tool called optical coherence tomography. ${ }^{23}$ In some affected individuals, the thinning is severe enough to cause holes in the retina that result in impaired vision. ${ }^{24}$

\section{Other Organ Systems}

In very rare cases, people with XLAS exhibit abnormalities that are not typical of the condition, due to the involvement of genes adjacent to the COL4A5 gene on the $\mathrm{X}$ chromosome. These so-called "contiguous gene syndromes" result from deletions of a chromosomal region that contains more than one gene. Deletion of portions of the COL4A5 gene and the adjacent COL4A6 gene are found in people with Alport syndrome who also display smooth muscle tumors of the esophagus, trachea, bronchi and female external genitalia, a condition known as diffuse leiomyomatosis. ${ }^{25}$ The dysregulation of smooth muscle cell proliferation that results in the formation of these tumors does not appear to be directly related to abnormalities of the $\alpha 345$ collagen IV scaffold, or of the scaffold formed by $\alpha 5$ and a 6 collagen IV chains (the $\alpha 556$ collagen IV scaffold). In extremely rare XY individuals the features of XLAS are associated with anomalies in the development of the face, cognitive deficits and abnormal red blood cell shape, resulting from combined deletion of the COL4A5 gene and several adjacent genes. ${ }^{26,27}$

As noted above, there is a third collagen IV species, the a556 collagen IV scaffold, that is found in some mammalian basement membranes, in addition to the previously mentioned $\alpha 345$ and $\alpha 112$ collagen IV scaffolds. The $\alpha 556$ collagen IV scaffold is normally found in the basement membranes that surround smooth muscles cells in the wall of the aorta. ${ }^{28,29}$ The $\alpha 556$ collagen IV scaffold is absent from aortic smooth muscle basement membranes in $\mathrm{XY}$ individuals with XLAS due to COL4A5 variants that prevent formation of the $\alpha 556$ collagen IV scaffold. This change in the structure of aortic smooth muscle membranes may in part explain abnormalities of the aorta, including aortic dilation, aortic dissection and aortic aneurysm in some XY individuals with XLAS. ${ }^{30,31}$

\section{Multidisciplinary Care of People with Alport Syndrome Primary Care Provider}

The primary care provider (PCP) plays a crucial role in the identification, diagnosis and management of people with Alport syndrome. The PCP (general pediatrician, family medicine provider, internist, nurse practitioner or physician's assistant) is typically the first health care professional to detect and perform initial assessment of key features of Alport syndrome such as hematuria and hearing loss, and directs referrals to nephrologists, audiologists, ophthalmologists and other specialty providers for further evaluation and management (Figure 1). The PCP also oversees the routine but critical components of preventative and well-child care including immunizations, parental counseling regarding dietary and developmental issues, age-appropriate screening studies and related issues. The PCP can reinforce messaging regarding recommended salt intake and maintaining body weight within ideal parameters.

When there is a known family history of Alport syndrome, the PCP can assist in identifying and evaluating atrisk family members. Urinalysis is an effective screening tool for Alport syndrome, because of the very high rates of hematuria in affected people $-100 \%$ in XY individuals with XLAS or ARAS, 95\% in XX individuals with XLAS and $50-75 \%$ in individuals with ADAS due to heterozygous variants in the COL4A3 or COL $4 A 4$ gene. ${ }^{11,12,32,33}$ A PCP familiar with the genetics of Alport syndrome can target at-risk family members for screening using urinalysis. For example, the $\mathrm{XX}$ offspring of an $\mathrm{XY}$ individual with XLAS will inherit the variant COL4A5 gene and will have hematuria, while the $X Y$ offspring will inherit the normal maternal COL4A5 gene and will not be affected. When an $\mathrm{XY}$ individual is diagnosed with Alport syndrome, the likelihood that the COL4A5 variant was inherited from their mother is $85-90 \%$ (there is a $10-15 \%$ incidence of spontaneous $C O L 4 A 5$ variants among XY individuals with XLAS), while the likelihood that the COL4A5 variant was inherited from their father is zero. Each XY or XX offspring of an XX individual with XLAS has a $50 \%$ probability of inheriting the variant COL $4 A 5$ gene. In the case of individuals with ARAS, each parent 


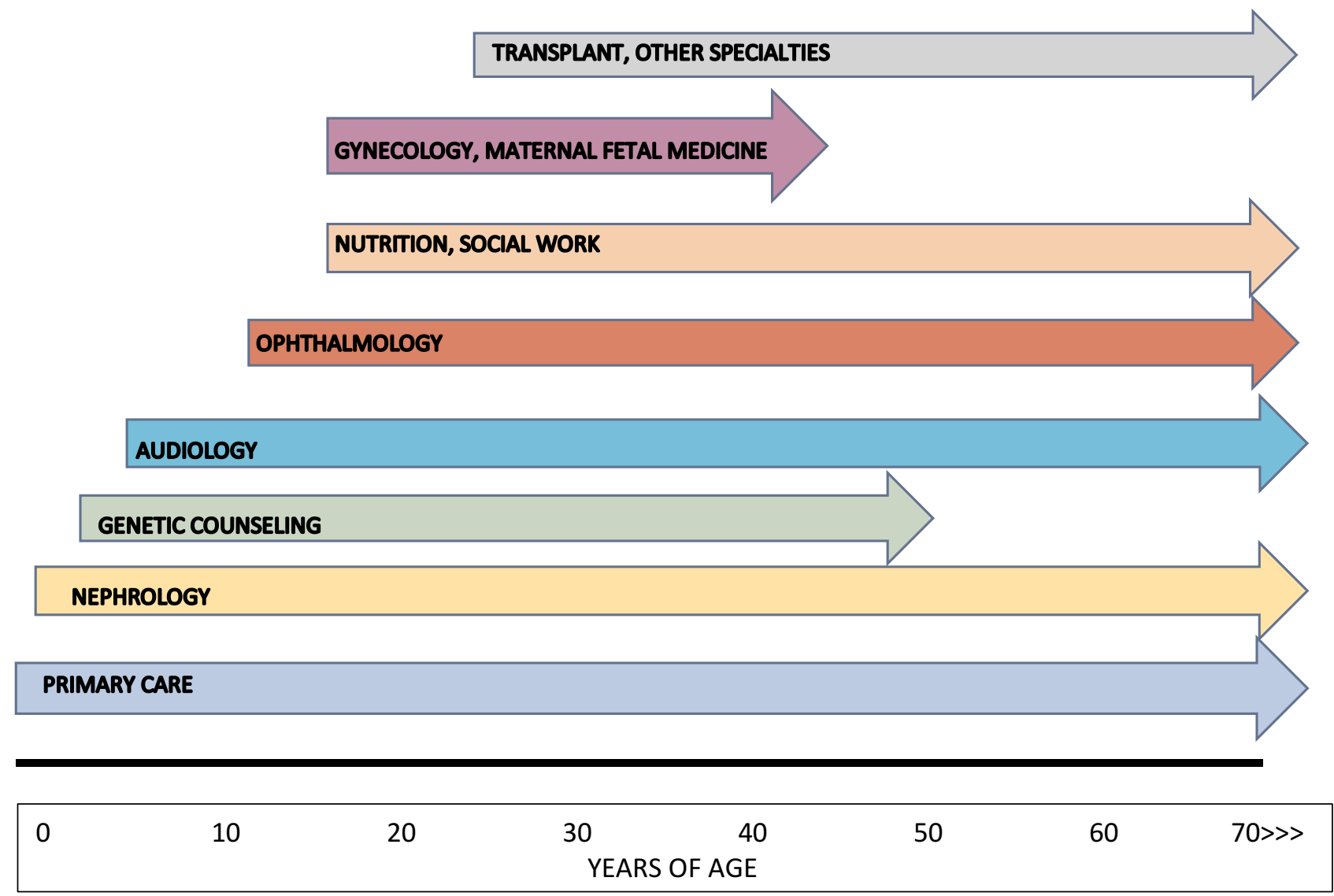

Figure I Multidisciplinary care for Alport syndrome - a timeline. Primary care providers become involved at birth and maintain their role in care throughout the life of the individual. Referral to nephrology should occur in the first I-2 years of life. Genetic counselors have an early role in diagnosis and subsequently in providing information on transmission of Alport syndrome to potential offspring. Audiology and ophthalmology frequently begin their clinical relationships with affected individuals in childhood and adolescence. The input of nutritionists and social workers becomes increasingly important as kidney disease progresses. Gynecologists assist in providing contraceptive guidance to $X X$ individuals receiving treatment with blockers of the renin-angiotensin-aldosterone system (RAAS) and, along with maternal-fetal medicine specialists, collaborate in the care of mothers with Alport syndrome during pregnancy, both before and after kidney transplantation. Transplant providers join the care team as affected individuals approach kidney failure and the need for kidney transplantation. Referral to cardiologists or other specialty providers may be necessary for some affected people.

typically exhibits a variant in one copy of the COL $4 A 3$ or COL4A4 gene, and is likely to display hematuria. With each pregnancy of a couple who both have a $C O L 4 A 3$ or COL $4 A 4$ variant there is a $50 \%$ risk of transmitting one of the variants, resulting in ADAS in the child, and a $25 \%$ risk of transmitting both variants, causing ARAS in the child.

When should the first urinalysis be performed in at-risk children with a known family history of Alport syndrome? Some infants and children with Alport syndrome will have episodes of tea- or cola-colored urine (macroscopic or gross hematuria), either spontaneously or associated with viral (typically upper respiratory) or bacterial infections, thereby effectively identifying themselves. However, hematuria in most infants and children with Alport syndrome is microscopic and requires urinalysis for detection. In children at-risk for XLAS or ARAS, urine collection using a urine bag should be performed by about one year of age. In children at risk for ADAS urine collection can wait until the child can void voluntarily, although earlier collection is not precluded. The diagnosis of Alport syndrome should be confirmed by genetic testing in those children and other family members who exhibit hematuria.

Alport syndrome is not currently included in neonatal screening for inherited disorders. When a family is transmitting known $C O L 4 A 3$, COL $4 A 4$ or $C O L 4 A 5$ variants, atrisk family members can undergo targeted gene sequencing as an alternative to urinalysis. Access to genetic testing may limit the application of this approach in some countries.

\section{Nephrology}

Pediatric and adult nephrologists provide care to people with Alport syndrome across the spectra of age (infancy to 
old age) and kidney function (normal to kidney failure). Nephrologists take part in the diagnosis of Alport syndrome and the evaluation of at-risk family members and are primary responsible for initiating and managing pharmacologic measures to slow kidney disease progression and delay kidney failure. Other tasks of the nephrologist include addressing the complications of advancing Alport kidney disease, including hypertension, salt and water retention, electrolyte disturbances such as hyperphosphatemia and hyperkalemia, altered vitamin D metabolism and anemia of chronic kidney disease. Ultimately, nephrologists coordinate planning for kidney replacement therapy (kidney transplantation and, when necessary, dialysis) and manage immunosuppression and other therapies following kidney transplantation.

The involvement of nephrology providers in the pediatric ( $0-18$ years) Alport population may depend on the local abundance and accessibility of nephrology services. Where nephrology resources are scarce, the PCP may perform the early diagnostic evaluation of children and adolescents who have hematuria, with or without a family history of Alport syndrome, and carry out monitoring for signs of progressive disease (albuminuria and proteinuria). Recent emphasis on very early pharmacologic intervention (discussed below) is likely to generate pressure to refer young people suspected of or confirmed to have Alport syndrome to nephrology at earlier ages. Telemedicine resources of various kinds provide a potential means to access nephrologists for the PCP in a nephrology-poor locale.

The last decade has seen a fairly rapid evolution of attitudes toward pharmacologic intervention to slow the progression of Alport kidney disease, from no intervention, to intervention at the time of appearance of albuminuria or proteinuria, to intervention at the time of diagnosis regardless of urinary albumin or protein levels. ${ }^{34-36}$ These developments have occurred against a backdrop of accumulating evidence that early intervention with inhibitors of the renin-angiotensin-aldosterone system, or RAAS, delays kidney failure in animals and people with Alport syndrome. ${ }^{9,37-40}$ Compared to providers of primary care to adult patients, providers of primary care to pediatric patients generally have relatively little experience with the use of inhibitors of the RAAS, such as angiotensin converting enzyme (ACE) inhibitors and angiotensin receptor blockers. Consequently, an emphasis an early intervention should lead to earlier involvement of nephrologists in the care of people with Alport syndrome, either directly or through technologies that facilitate remote consultation and management. Nephrologists are likely to have new agents to consider as add-on treatments for Alport kidney disease in the relatively near future. ${ }^{41-43}$

Nephrologists, particularly those practicing in large medical centers, may have more ready access to genetic diagnostic and counseling services than PCPs in small, or even larger, multispecialty practices. Clinics devoted to the diagnosis and in some instances management of genetic kidney diseases potentially provide a relatively smooth approach to genetic diagnosis of Alport syndrome. The role of genetic counseling in Alport syndrome care is discussed further below.

Education of families about Alport syndrome, including providing accurate prognostic information, is a critical task that typically falls to nephrologists who, regardless of their personal knowledge of the condition, are bestpositioned to carry out this responsibility. Fortunately, there are numerous educational resources on-line available to nephrologists as well as other providers of care to people with Alport syndrome (for example, alportsyndrome.org). Resources written specifically for nonmedical professionals are also easily accessible.

\section{Genetics and Genetic Counseling}

The value of the contributions of genetic counselors to the diagnosis of Alport syndrome, and other genetic kidney disorders, in index cases and their families is difficult to overstate. Genetic counselors who develop a focus on genetic kidney disorders are at least as knowledgeable about these conditions as the nephrologists they work with, and may indeed by better informed. Genetic counselors are skilled in navigating the challenges presented by the multitude of health insurance programs and their varying coverage of genetic testing. Genetic counselors are familiar with the range of genetic testing tools available, from highly focused to broad-based next generation sequencing panels, as well as whole exome sequencing, and assist the nephrologist in selecting the most appropriate tool for each patient and family. Genetic counselors have expertise in the explanation of genetic testing results to individuals and families, identifying at-risk family members who can be offered targeted testing after identification of a variant in a family, and providing information relevant to reproductive planning.

Unfortunately, there is a mismatch between the supply of genetic counselors and the demand for their services. Telemedicine provides a means for providing remote 
genetic counseling services. Commercial genetic testing laboratories are increasingly offering genetic counseling as part of their testing packages.

When clinical features and family history are highly suggestive of a diagnosis of Alport syndrome, formal consultation by a genetics physician before ordering genetic testing may not be necessary. Genetics consultation is especially valuable when the available clinical and pedigree information is consistent with a relatively long list of potential diagnoses. For example, Alport syndrome is only one of many conditions in which kidney disease can be associated with hearing loss. ${ }^{44} \mathrm{~A}$ broad next generation sequencing panel or whole exome sequencing may be more appropriate when Alport syndrome is just one of a number of potential diagnoses. Broad panels and whole exome sequencing frequently identify variants in multiple genes that may or may not be pathogenic, may or may not be relevant to the reason for testing, and which may strain the nephrologist's capacity to provide appropriate counseling and guidance. For these reasons, consultation by a geneticist is advisable when clinical and pedigree information creates diagnostic uncertainty.

\section{Audiology}

Formal hearing evaluation by an audiologist (ideally a pediatric audiologist) should be initiated at 5-6 years in XY children with XLAS and children with ARAS. This recommendation is based on natural history data showing that about a third of XY children with XLAS have detectable hearing loss by age 10 , rising to $60 \%$ by age 20 , and that about $20 \%$ of children with ARAS have hearing loss by age $10 .^{11,45}$ The PCP or nephrologist should consider earlier referral to audiology for affected children with overt proteinuria, children who fail hearing screens or children in whom attainment of speech milestones is delayed.

XX individuals with XLAS have a relatively low lifetime risk of hearing loss of about $30 \%$, with the hearing loss occurring in $10 \%$ by age $40 .{ }^{12}$ Those with proteinuria or declining kidney function are more likely to have hearing loss. ${ }^{11,12}$ Audiology referral is recommended for XX individuals with XLAS who have proteinuria or reduced kidney function, and for those who fail a hearing screen or complain or exhibit signs of decreased hearing. Hearing loss occurs in less than $20 \%$ of people with ADAS, so evaluation by audiology is recommended when there is suspicion of hearing loss. ${ }^{18,46}$
Identification of individuals who would benefit from hearing aids, along with the selection and fitting of devices and education in their use are all tasks that are ideally performed by audiologists. One of the major barriers to successful intervention with hearing aids is the reluctance of children and adolescents to use them regularly. Audiologists who have experience working with the pediatric population can help these individuals derive the maximum benefit from their devices. Audiologists are accustomed to discussing with their clients the risks of noise-induced injury, which likely contributes to hearing loss in people with Alport syndrome. Audiologists are also in the best position to counsel individuals and families about the costs of various devices. This is very important because private health insurance programs, at least in the United States, generally do not cover the costs of hearing aids. Medicaid covers the costs of hearing aids for children nationally, while coverage for adults varies by state. It should be noted that over-the-counter hearing aids are not the optimal option for people with Alport syndrome, due to their limited features and non-specific fitting.

Formal consultation by an otolaryngologist is not generally necessary for people with hearing loss due to Alport syndrome, unless there are congenital anomalies of the ears or there are acquired problems such as recurrent ear infections. Hearing loss in Alport syndrome rarely progresses to the point where hearing aids are ineffective, and affected individuals retain the ability to distinguish the various sounds of speech (speech discrimination), so cochlear implantation is rarely, if ever, indicated.

\section{Ophthalmology}

In people with Alport syndrome, the major threats to normal vision are anterior lenticonus and holes in the macular region of the retina. In people with anterior lenticonus, refractive errors caused by the abnormally shaped lens can be ameliorated with corrective lenses. Cataracts that form as a result of anterior lenticonus can be addressed surgically. People with Alport syndrome can develop other ocular problems, such as glaucoma, that are not specific to Alport syndrome but can further impair vision in affected individuals.

Regular ophthalmologic examinations to assess general ocular health and to screen for anterior lenticonus should begin in adolescence for XY individuals with XLAS due to COL4A5 variants that prevent synthesis of a full-length $\alpha 5$ collagen IV chain and in people with ARAS. Earlier examination should occur if there is evidence of decreased 
visual acuity. XX individuals with XLAS and people with ADAS should undergo ophthalmologic examination if there is concern regarding visual deficits.

Ophthalmological assessment can also be useful in the diagnosis of Alport syndrome, since anterior lenticonus and dot-fleck retinopathy are specific to the condition. Some individuals with Alport syndrome experience recurrent corneal erosions or abrasions, likely due to abnormalities of the collagen composition of corneal basement membranes, and may need guidance in regards to protective eyewear to reduce the frequency of erosions.

\section{Transplant Medicine and Surgery}

The cumulative lifetime risk of kidney failure is close to $100 \%$ for XY persons with XLAS and for people with ARAS, $30-40 \%$ for XX persons with XLAS and possibly $10-20 \%$ of those with ADAS, although this latter percentage is less certain. Thus, many people with Alport syndrome will encounter transplant providers at some point during their lives.

The primary outcome measures of kidney transplant success are survival of the recipient and the duration of transplant function following the procedure. Both of these outcomes are excellent for people with Alport syndrome. ${ }^{47,48}$ In contrast to other causes of kidney failure, such as diabetic kidney disease, Alport syndrome is not typically associated with severe cardiovascular disease, a major contributor to death in the years after kidney transplantation in people with diabetes. Alport syndrome does not return in the kidney transplant, unlike focal segmental glomerulosclerosis, which frequently recurs in kidney transplant and often leads to failure of the transplant. Consequently, pre-transplant preparation for kidney transplant and care following the transplant are fairly routine. However, there are two exceptions to this rule that transplant medicine and surgery providers must consider as they prepare people with Alport syndrome for transplant and care for them following transplant.

First, the assessment and selection of living kidney donors for people with Alport syndrome must be informed by an understanding of the genetics of Alport syndrome, with the goal of avoiding the removal of a kidney from a person with a variant in the COL4A3, COL4A4 or COL4A5 gene. $^{49}$ In the best-case scenario, any at-risk related individual is screened for the variant or variants identified in the prospective kidney transplant recipient, and those individuals who share a variant with the recipient are excluded from consideration as kidney donors.
Genetic counselors should be involved in the donor evaluation process. Recommendations for evaluation and selection of living donors for people with Alport syndrome have been promulgated ${ }^{49}$ but at this time there is not a consensus on such recommendations.

Second, certain people with Alport syndrome, namely XY individuals with XLAS and persons with ARAS, are at risk for a unique complication of kidney transplant known as post-transplant antibody-mediated glomerulonephritis. ${ }^{49}$ In this condition, the recipient forms antibodies to the $\alpha 345$ collagen IV network of the glomerular basement membranes of the kidney transplant, resulting in severe inflammation of the transplant that is difficult to treat effectively. Fortunately, this is a very unusual event that occurs in less than $5 \%$ of people with Alport syndrome who undergo kidney transplantation. At-risk individuals can be monitored for antibodies to the $\alpha 345$ collagen IV network using blood tests. Kidney transplant recipients typically undergo frequent blood testing to detect changes in kidney transplant function, and recipients who exhibit decreased transplant function frequently undergo biopsy of the kidney transplant to determine the cause of dysfunction. Biopsy of the kidney transplant should be performed promptly in recipients who have Alport syndrome and decreased transplant function, or who have evidence of antibodies to the $\alpha 345$ collagen IV network on blood tests, to confirm a suspected diagnosis of post-transplant antibody-mediated glomerulonephritis and initiate appropriate treatment.

\section{Gynecology, Obstetrics and Maternal-Fetal Medicine}

Chronic kidney diseases, including Alport syndrome, increase the risks of pregnancy to both the mother and the fetus. ${ }^{50}$ These risks include preeclampsia, premature delivery, low birth weight, cesarean section and failure of the pregnancy. The likelihood of these complications is higher in mothers with reduced kidney function, elevated urine protein levels or poorly controlled hypertension. ${ }^{51}$ Kidney function that is normal or only mildly reduced at the start of pregnancy usually remains stable during pregnancy; however when kidney function is significantly abnormal at the start of pregnancy it may decrease further during pregnancy. ${ }^{52}$ Although there are several reports describing pregnancy complications in mothers with Alport syndrome, ${ }^{50,53,54}$ there is no evidence as yet that the risk of complications is higher in those with Alport 
syndrome compared to other causes of chronic kidney disease.

Effective contraception to prevent unintended pregnancy is an important part of the care of XX individuals with Alport syndrome, in order to avoid injury to the heart and kidneys of a developing fetus from treatment of the mother with RAAS blockers, and to allow a full discussion of the risks of pregnancy and facilitate planning for prenatal care that is appropriate for the mother's stage of chronic kidney disease. ${ }^{55}$ The choice of contraceptive method should be guided by providers familiar with the use of contraceptive agents in those with chronic kidney disease. Mothers with Alport syndrome should be closely monitored during pregnancy; those with kidney function less than about $60 \%$ of normal, or those with proteinuria or hypertension, require more intensive follow-up from nephrologists as well as maternal-fetal medicine specialists who specialize in the care of mothers who are at increased risk for pregnancy complications. Following kidney transplantation, nephrologists, transplant providers and maternal-fetal medicine specialists may all be involved in prenatal counseling and pregnancy care.

\section{Other Specialties}

Some people with Alport syndrome may choose to pursue in vitro fertilization and pre-implantation diagnosis, involving specialty care in these procedures. The smooth muscle tumors that occur in rare individuals with XLAS and diffuse leiomyomatosis may require the involvement of gastroenterology, gastrointestinal surgery, pulmonology, thoracic surgery and/or gynecology.

There are currently no guidelines or recommendations for the evaluation and management of aortic disease in people with Alport syndrome. Echocardiography will reveal dilation and other abnormalities of the thoracic aorta and is used to monitor cardiac structure and health in adolescents and young adults with chronic kidney disease, especially if they are receiving antihypertensive medications. Echocardiography is also frequently performed when people are initiated on dialysis or as part of preparation for kidney transplantation. Cardiology should be consulted when echocardiography reveals abnormalities of the aorta. Echocardiography will not detect changes in the abdominal portion of the aorta.

\section{Allied Professionals}

The active involvement of dietary professionals with expertise in the counseling and management of people with chronic kidney disease is essential to optimal care of people with Alport syndrome. In early stages of Alport kidney disease, while kidney function is still normal, control of dietary salt intake contributes to the efficacy of medications that inhibit RAAS activity. Many people, including children, consume significantly more salt than they require, and stand to benefit from dietary changes that reduce their salt intake toward recommended levels. Accommodation to recommended salt intake can also make it easier to further limit salt intake at more advanced stages of Alport kidney disease, when hypertension frequently becomes a significant issue. As kidney disease progresses in people with Alport syndrome, kidney dieticians provide invaluable assistance in evaluation and guidance of intake of other dietary components such as calcium, phosphorus and protein.

People with Alport syndrome and their families face emotional and financial stresses that frequently challenge the capacities of primary and specialty medical providers. Social workers assist families in obtaining financial support to which they are entitled, and can assist in identifying signs of emotional stress. The support provided by social workers becomes increasingly important as Alport kidney disease progresses to kidney failure and the need for dialysis and/or kidney transplantation. The involvement of psychiatry or psychology may be indicated when emotional stress interferes with medical care or other areas of life, including school, work and family.

\section{Conclusions}

The pursuit of optimal clinical outcomes in people with Alport syndrome relies on early diagnosis and comprehensive management, and entails the participation of professionals with a variety of skills. Coordination of care typically falls to the primary care provider or the nephrologist. People with Alport syndrome lead fulfilling and productive lives that are enhanced when their care providers rise to this challenge.

\section{Acknowledgments}

Over the course of a three-decade career, I have met hundreds of people with Alport syndrome around the globe who have shared their stories with me. I have encountered and collaborated with dozens of clinicians and scientists of astonishing skill and dedication who are working to improve the diagnosis and treatment of this condition. I thank all of them for teaching me about Alport syndrome. 
In many people with Alport syndrome, the genetic abnormality involves a gene on the $\mathrm{X}$ chromosome. Consequently, in any comprehensive discussion of Alport syndrome it is impossible to ignore chromosomal sex (XY or $\mathrm{XX}$ ), since chromosomal sex can have a profound impact on the course and outcome of the condition. However, chromosomal sex and gender identity are not synonymous. In this paper I have tried to use gender-neutral terms, such as XY or $\mathrm{XX}$ individual, rather than gender-specific terms such as boy, man or male and girl, woman or female. I appreciate the reader's indulgence. I have attempted to use plain language with the goal of making this paper accessible to a wide audience. Clifford Kashtan: he, his, him.

\section{Disclosure}

Dr. Kashtan is Co-Director of ASTOR, the Alport Syndrome Treatments and Outcomes Registry (ClinicalTrials.gov Identifier NCT00481130), which has received support from the Alport Syndrome Foundation and the Schuman and Petersen families. Dr. Kashtan was a consultant and later a site investigator for the CARDINAL trial of bardoxolone-methyl, sponsored by Reata Pharmaceuticals (ClinicalTrials.gov Identifier NCT03019185). Dr. Kashtan was a consultant and is a site investigator for the HERA trial of lademirsen, sponsored by Sanofi-Genzyme (ClinicalTrials.gov Identifier NCT02855268). Dr. Kashtan has recent consulting relationships with ONO Pharmaceuticals, DaiichiSankyo, Travere Therapeutics, BridgeBio, METiS Pharmaceuticals and Boehringer-Ingelheim. The author reports no other conflicts of interest related to this work.

\section{References}

1. Quinlan C, Rheault MN. Genetic basis of type IV collagen disorders of the kidney. Clin J Am Soc Nephrol. 2021;CJN.19171220. doi:10.2215/ CJN.19171220

2. Kashtan CE, Kim Y. Distribution of the alpha 1 and alpha 2 chains of collagen IV and of collagens V and VI in Alport syndrome. Kidney Int. 1992;42(1):115-126. doi:10.1038/ki.1992.269

3. Kalluri R, Shield CF, Todd P, Hudson BG, Neilson EG. Isoform switching of type IV collagen is developmentally arrested in X-linked Alport syndrome leading to increased susceptibility of renal basement membranes to endoproteolysis. J Clin Invest. 1997;99 (10):2470-2478. doi:10.1172/JCI119431

4. Kashtan CE, Kim Y, Lees GE, Thorner PS, Virtanen I, Miner JH. Abnormal glomerular basement membrane laminins in murine, canine, and human Alport syndrome: aberrant laminin alpha2 deposition is species independent. $J$ Am Soc Nephrol. 2001;12(2):252-260. doi:10.1681/ASN.V122252

5. Meehan DT, Delimont D, Cheung L, et al. Biomechanical strain causes maladaptive gene regulation, contributing to Alport glomerular disease. Kidney Int. 2009;76(9):968-976. doi:10.1038/ki.2009.324
6. Rumpelt HJ. Hereditary nephropathy (Alport syndrome): correlation of clinical data with glomerular basement membrane alterations. Clin Nephrol. 1980;13(5):203-207.

7. Gubler M, Levy M, Broyer M, et al. Alport's syndrome. A report of 58 cases and a review of the literature. Am J Med. 1981;70 (3):493-505. doi:10.1016/0002-9343(81)90571-4

8. Liapis H, Foster K, Miner JH. Red cell traverse through thin glomerular basement membrane. Kidney Int. 2002;61(2):762-763. doi:10.1046/j.1523-1755.2002.00181.x

9. Gross O, Tönshoff B, Weber LT, et al. A multicenter, randomized, placebo-controlled, double-blind Phase 3 trial with open-arm comparison indicates safety and efficacy of nephroprotective therapy with ramipril in children with Alport's syndrome. Kidney Int. 2020;97 (6):1275-1286. doi:10.1016/j.kint.2019.12.015

10. Kashtan CE, Gubler MC, Sisson-Ross S, Mauer M. Chronology of renal scarring in males with Alport syndrome. Pediatr Nephrol. 1998;12(4):269-274. doi:10.1007/s004670050451

11. Jais JP, Knebelmann B, Giatras I, et al. X-linked Alport syndrome: natural history in 195 families and genotype- phenotype correlations in males. J Am Soc Nephrol. 2000;11(4):649-657. doi:10.1681/ASN. V114649

12. Jais JP, Knebelmann B, Giatras I, et al. X-linked Alport syndrome: natural history and genotype-phenotype correlations in girls and women belonging to 195 families: a "European Community Alport Syndrome Concerted Action" Study. J Am Soc Nephrol. 2003;14 (10):2603-2610. doi:10.1097/01.ASN.0000090034.71205.74

13. Bekheirnia MR, Reed B, Gregory MC, et al. Genotype-phenotype correlation in X-linked Alport syndrome. J Am Soc Nephrol. 2010;21 (5):876-883. doi:10.1681/ASN.2009070784

14. Horinouchi T, Nozu K, Yamamura T, et al. Detection of splicing abnormalities and genotype-phenotype correlation in X-linked Alport syndrome. J Am Soc Nephrol. 2018;29(8):2244-2254. doi:10.1681/ASN.2018030228

15. Cosgrove D, Samuelson G, Pinnt J. Immunohistochemical localization of basement membrane collagens and associated proteins in the murine cochlea. Hear Res. 1996;97(1-2):54-65. doi:10.1016/S03785955(96)80007-4

16. Zehnder AF, Adams JC, Santi PA, et al. Distribution of type IV collagen in the cochlea in Alport syndrome. Arch Otolaryngol Head Neck Surg. 2005;131(11):1007-1013. doi:10.1001/archotol.1 31.11.1007

17. Imafuku A, Nozu K, Sawa N, et al. Autosomal dominant form of type IV collagen nephropathy exists among patients with hereditary nephritis difficult to diagnose clinicopathologically. Nephrology (Carlton). 2018;23(10):940-947. doi:10.1111/nep.13115

18. Marcocci E, Uliana V, Bruttini M, et al. Autosomal dominant Alport syndrome: molecular analysis of the COL4A4 gene and clinical outcome. Nephrol Dial Transplant. 2009;24(5):1464-1471. doi:10.1093/ndt/gfn681

19. Cheong HI, Kashtan CE, Kim Y, Kleppel MM, Michael AF. Immunohistologic studies of type IV collagen in anterior lens capsules of patients with Alport syndrome. Lab Invest. 1994;70 (4):553-557.

20. Kleppel MM, Kashtan C, Santi PA, Wieslander J, Michael AF. Distribution of familial nephritis antigen in normal tissue and renal basement membranes of patients with homozygous and heterozygous Alport familial nephritis. Relationship of familial nephritis and goodpasture antigens to novel collagen chains and type IV collagen. Lab Invest. 1989;61(3):278-289.

21. Kleppel MM, Fan WW, Cheong HI, Kashtan CE, Michael AF. Immunochemical studies of the Alport antigen. Kidney Int. 1992;41 (6):1629-1637. doi:10.1038/ki.1992.235

22. Savige J, Sheth S, Leys A, Nicholson A, Mack HG, Colville D. Ocular features in Alport syndrome: pathogenesis and clinical significance. Clin J Am Soc Nephrol. 2015;10(4):703-709. doi: $10.2215 /$ CJN. 10581014 
23. Ahmed F, Kamae KK, Jones DJ, et al. Temporal macular thinning associated with X-linked Alport syndrome. JAMA Ophthalmol. 2013;131(6):777-782. doi:10.1001/jamaophthalmol.2013.1452

24. Rahman W, Banerjee S. Giant macular hole in Alport syndrome. Can J Ophthalmol. 2007;42(2):314-315.

25. Antignac C, Zhou J, Sanak M, et al. Alport syndrome and diffuse leiomyomatosis: deletions in the $5^{\prime}$ end of the COL4A5 collagen gene. Kidney Int. 1992;42(5):1178-1183. doi:10.1038/ki.1992.402

26. Jonsson JJ, Renieri A, Gallagher PG, et al. Alport syndrome, mental retardation, midface hypoplasia, and elliptocytosis: a new X linked contiguous gene deletion syndrome? J Med Genet. 1998;35 (4):273-278. doi:10.1136/jmg.35.4.273

27. Rodriguez JD, Bhat SS, Meloni I, et al. Intellectual disability, midface hypoplasia, facial hypotonia, and Alport syndrome are associated with a deletion in Xq22.3. Am J Med Genet A. 2010;152A (3):713-717. doi:10.1002/ajmg.a.33208

28. Borza DB, Bondar O, Ninomiya Y, et al. The NC1 domain of collagen IV encodes a novel network composed of the alpha 1, alpha 2, alpha 5, and alpha 6 chains in smooth muscle basement membranes. J Biol Chem. 2001;276(30):28532-28540. doi:10.1074/ jbc.M103690200

29. Seki T, Naito I, Oohashi T, Sado Y, Ninomiya Y. Differential expression of type IV collagen isoforms, alpha5(IV) and alpha6(IV) chains, in basement membranes surrounding smooth muscle cells. Histochem Cell Biol. 1998;110(4):359-366. doi:10.1007/s004180050296

30. Kashtan CE, Segal Y, Flinter F, Makanjuola D, Gan JS, Watnick T. Aortic abnormalities in males with Alport syndrome. Nephrol Dial Transplant. 2010;25(11):3554-3560. doi:10.1093/ndt/gfq271

31. Patel J, Abt P, Cheng K, Aurigemma G, Rosenthal L. Type A dissection in a patient with Alport syndrome. Circ Cardiovasc Imaging. 2020;13(12):e010701. doi:10.1161/ CIRCIMAGING.120.010701

32. Boye E, Mollet G, Forestier L, et al. Determination of the genomic structure of the COL4A4 gene and of novel mutations causing autosomal recessive Alport syndrome. Am J Hum Genet. 1998;63 (5):1329-1340. doi:10.1086/302106

33. Heidet L, Arrondel C, Forestier L, et al. Structure of the human type IV collagen gene COL4A3 and mutations in autosomal Alport syndrome. J Am Soc Nephrol. 2001;12(1):97-106. doi:10.1681/ ASN.V12197

34. Kashtan CE, Ding J, Gregory M, et al. Clinical practice recommendations for the treatment of Alport syndrome: a statement of the Alport syndrome research collaborative. Pediatr Nephrol. 2013;28 (1):5-11. doi:10.1007/s00467-012-2138-4

35. Kashtan CE, Gross O. Clinical practice recommendations for the diagnosis and management of Alport syndrome in children, adolescents, and young adults-an update for 2020. Pediatr Nephrol. 2020;36(3):711-719. doi:10.1007/s00467-020-04819-6

36. Savige J, Gregory M, Gross O, Kashtan C, Ding J, Flinter F. Expert guidelines for the management of Alport syndrome and thin basement membrane nephropathy. $J$ Am Soc Nephrol. 2013;24 (3):364-375. doi:10.1681/ASN.2012020148

37. Gross O, Beirowski B, Koepke ML, et al. Preemptive ramipril therapy delays renal failure and reduces renal fibrosis in col4a3-knockout mice with Alport syndrome. Kidney Int. 2003;63(2):438-446. doi:10.1046/j.1523-1755.2003.00779.x

38. Gross O, Licht C, Anders HJ, et al. Early angiotensin-converting enzyme inhibition in Alport syndrome delays renal failure and improves life expectancy. Kidney Int. 2012;81(5):494-501. doi:10.1038/ki.2011.407
39. Temme J, Peters F, Lange K, et al. Incidence of renal failure and nephroprotection by RAAS inhibition in heterozygous carriers of $\mathrm{X}$-chromosomal and autosomal recessive Alport mutations. Kidney Int. 2012;81(8):779-783. doi:10.1038/ki.2011.452

40. Yamamura T, Horinouchi T, Nagano C, et al. Genotype-phenotype correlation and the effects of treatment with angiotensin-targeting drugs in Japanese patients with male X-linked Alport syndrome. Kidney Int. 2020;in press.

41. Omachi K, Miner JH. Alport syndrome therapeutics: ready for prime-time players. Trends Pharmacol Sci. 2019;40(11):803-806. doi:10.1016/j.tips.2019.07.012

42. Torra R, Furlano M. New therapeutic options for Alport syndrome. Nephrol Dial Transplant. 2019;34(8):1272-1279. doi:10.1093/ndt/ gfz131

43. Mabillard H, Sayer JA. SGLT2 inhibitors - a potential treatment for Alport syndrome. Clin Sci (Lond). 2020;134(4):379-388. doi:10.1042/CS20191276

44. Phelan PJ, Rheault MN. Hearing loss and renal syndromes. Pediatr Nephrol. 2018;33(10):1671-1683. doi:10.1007/s00467-017-3835-9

45. Oka M, Nozu K, Kaito H, et al. Natural history of genetically proven autosomal recessive Alport syndrome. Pediatr Nephrol. 2014;29 (9):1535-1544. doi:10.1007/s00467-014-2797-4

46. Kamiyoshi N, Nozu K, Fu XJ, et al. Genetic, clinical, and pathologic backgrounds of patients with autosomal dominant Alport syndrome. Clin J Am Soc Nephrol. 2016;11(8):1441-1449. doi:10.2215/ CJN.01000116

47. Temme J, Kramer A, Jager KJ, et al. Outcomes of male patients with Alport syndrome undergoing renal replacement therapy. Clin $\mathrm{J} \mathrm{Am}$ Soc Nephrol. 2012;7(12):1969-1976. doi:10.2215/CJN.02190312

48. Kashtan CE, McEnery PT, Tejani A, Stablein DM. Renal allograft survival according to primary diagnosis: a report of the North American Pediatric Renal Transplant Cooperative Study. Pediatr Nephrol. 1995;9(6):679-684. doi:10.1007/BF00868709

49. Kashtan CE. Renal transplantation in patients with Alport syndrome: patient selection, outcomes, and donor evaluation. Int J Nephrol Renovasc Dis. 2018;11:267-270. doi:10.2147/IJNRD.S150539

50. Yefet E, Tovbin D, Nachum Z. Pregnancy outcomes in patients with Alport syndrome. Arch Gynecol Obstet. 2016;293(4):739-747. doi:10.1007/s00404-015-3893-9

51. Piccoli GB, Attini R, Vasario E, et al. Pregnancy and chronic kidney disease: a challenge in all CKD stages. Clin J Am Soc Nephrol. 2010;5(5):844-855. doi:10.2215/CJN.07911109

52. Hui D, Hladunewich MA. Chronic kidney disease and pregnancy. Obstet Gynecol. 2019;133(6):1182-1194. doi:10.1097/ AOG.0000000000003256

53. Brunini F, Zaina B, Gianfreda D, et al. Alport syndrome and pregnancy: a case series and literature review. Arch Gynecol Obstet. 2018;297(6):1421-1431. doi:10.1007/s00404-018-4720-х

54. Crovetto F, Moroni G, Zaina B, Acaia B, Ossola MW, Fedele L. Pregnancy in women with Alport syndrome. Int Urol Nephrol. 2013;45(4):1223-1227. doi:10.1007/s11255-012-0154-8

55. Savige J, Colville D, Rheault M, et al. Alport syndrome in women and girls. Clin J Am Soc Nephrol. 2016;11(9):1713-1720. doi:10.2215/CJN.00580116 


\section{Publish your work in this journal}

The Journal of Multidisciplinary Healthcare is an international, peerreviewed open-access journal that aims to represent and publish research in healthcare areas delivered by practitioners of different disciplines. This includes studies and reviews conducted by multidisciplinary teams as well as research which evaluates the results or conduct of such teams or healthcare processes in general. The journal

covers a very wide range of areas and welcomes submissions from practitioners at all levels, from all over the world. The manuscript management system is completely online and includes a very quick and fair peer-review system. Visit http://www.dovepress.com/testimonials. php to read real quotes from published authors. 